\title{
Research and comparative analysis of methods linear interpolation trajectory of the working tool of a two-tier mechanism
}

\author{
Ivan Naumov ${ }^{1, *}$, Anton Paschenko. ${ }^{1}$ and Aleksandr Koltcov. ${ }^{1}$ \\ ${ }^{1}$ Don state technical university (DSTU), Department of Radioelectronic and electrotechnical systems and complexes, 344002, Rostov-on- \\ Don, Russia
}

\begin{abstract}
In this paper the comparative analysis of the interpolation methods (Bresenham's method and the least distance method) of the linear interpolation trajectory of the working tool of a two-tier mechanism is performed and its error is examined. The kinematic diagram of the two-tier mechanism is studied; the direct and inverse kinematics are determined. To perform the comparative analysis, the method of the mechanism's interpolation grid construction is studied.
\end{abstract}

\section{Introduction}

In most cases the flattened materials (metal, fabric, leather, stone and etc.) profiling operation by using the firmware reduces to segmentation (division) of the figure's compound contour to elementary figures, which can most be the rectilinear segment, considering the interpolation algorithms implementation simplicity. When dividing a compound contour to rectilinear segments the processing accuracy of a raw workpiece is determined by the number and the accuracy of the interpolation method applied.

The problem of an optimal compound contour division to the rectilinear segments is solved by most electric accounting machines (systems) and the interpolation problem rests to a $\mathrm{CNC}$ devices, which solution accuracy depends on its kinematic aspects, operating mechanism's accuracy and the interpolation methods.

There are many different methods of the linear interpolation, such as digital differential analyzer (DDA) and Bresenham's method [1], which are the most known methods at the present time.

The choice of the particular method to solve the linear interpolation problem depends on the kinematic characteristics of the equipment.

Due to this, here, we perform the comparative analysis of the rectilinear segment interpolation methods to study the interpolation error for every method.

It is known, that the DDA is more complicated in a way of hardware implementation, since it involves the recurrent division operations, so as the comparative methods in this work the Bresenham's method is regarded, as well as the method for the two-tier mechanism, suggested before [2].

\section{Two-tier mechanism}

The two-tier mechanism, which kinematic diagram is illustrated in fig. 1, is represented as the disk with radius $\mathrm{R}$, which can turn around the center, located at coordinate origin of the basic coordinate system XOY through an angle, where the rotation angle $0 \leq \alpha<2 \pi$.

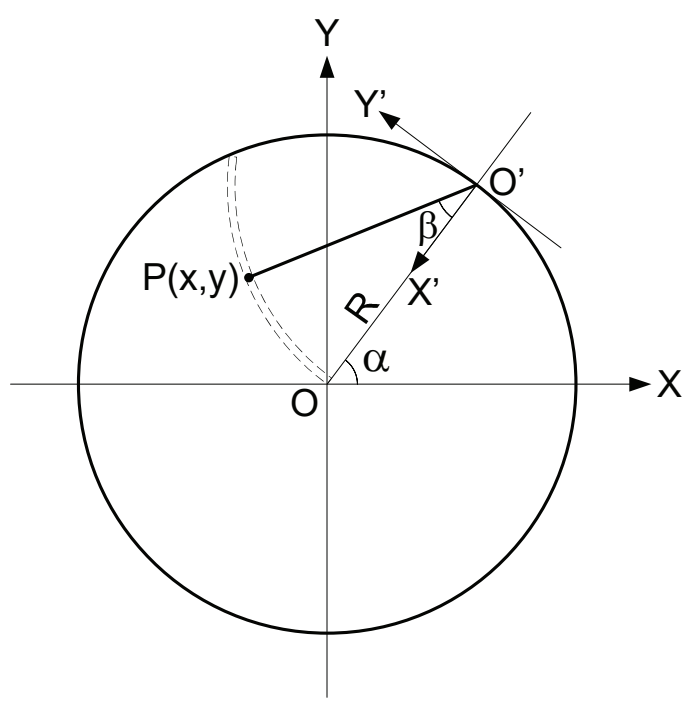

Fig.1. The kinematic diagram of the two-tier mechanism

At the edge of the disk the rod is fixed and its length equals to disk's radius $R$. The rod can turn through angle $\beta$ on itself, which axis is located in the additional coordinate system $X^{\prime} O^{\prime} Y^{\prime}$, where the rod's rotation angle $0 \leq \beta \leq \frac{\pi}{3}$. The working tool is fixed on the edge of the rod at the point $P(x, y)$ and goes through the disk, that's why disk is provided with a slot of the certain width. The slot strikes an arc of the circle with the radius $R$ and the

\footnotetext{
* Corresponding author: naumov_ivan85@mail.ru
} 
center in the origin of additional coordinate system $X^{\prime} O^{\prime} Y^{\prime}$.

\section{The solution of the inverse kinematic problem}

The solution of an inverse kinematic problem [2] can be described as following:

$$
\left\{\begin{array}{c}
\alpha(t)=\arcsin \left(\frac{x(t) \cdot \cos \beta(t)-x(t)-\sin \beta(t) \cdot y(t)}{2 \cdot R(\cos \beta(t)+1)}\right) \\
\beta(t)=\arccos \left(\frac{-x^{2}(t)-y^{2}(t)+2 \cdot R^{2}}{2 \cdot R^{2}}\right)
\end{array}\right.
$$

The solution of the direct kinematic problem can be written in accordance with [2] as following:

$$
\left\{\begin{array}{l}
x(t)=R \cdot \sin (\beta(t)+\alpha(t))-R \cdot \sin \alpha(t) \\
y(t)=R \cdot \cos (\beta(t)+\alpha(t))-R \cdot \cos \alpha(t)
\end{array}\right.
$$

When solved the direct kinematics for the two-tier mechanism considering the angle resolution of the disk rotation $\Delta \alpha$ and the rod rotation angle resolution $\Delta \beta$, all connection joints of the interpolation grid coordinates can be defined. For this purpose, in MATLAB development environment the virtual prototype was actualized. The prototype allows calculating coordinates of all connection joints in the interpolation grid of the two-tier mechanism. The input parameters of the prototype are: disk radius $R$ and the discrete pitch size of angles $\Delta \alpha$ and $\Delta \beta$. In accordance with the prototype's input parameters its algorithm creates a rectangular matrix of the joint coordinates $M$ expressed in units $n \times m$, which has the following form:

$$
\begin{aligned}
& M(i, j)= \\
& \left(\alpha_{1} ; \beta_{1}\right) \quad\left(\alpha_{2} ; \beta_{1}\right) \quad \ldots \quad\left(\alpha_{j} ; \beta_{1}\right) \quad\left(\alpha_{j+1} ; \beta_{1}\right) \\
& \begin{array}{lllll}
\left(\alpha_{1} ; \beta_{2}\right) & \left(\alpha_{2} ; \beta_{2}\right) \quad \ldots & \left(\alpha_{j} ; \beta_{2}\right) & \left(\alpha_{j+1} ; \beta_{2}\right)
\end{array} \\
& \begin{array}{ccccc}
\cdots & \cdots & \cdots & \cdots & \cdots \\
\left(\alpha_{1} ; \beta_{i}\right) & \left(\alpha_{2} ; \beta_{i}\right) & \cdots & \left(\alpha_{j} ; \beta_{i}\right) & \left(\alpha_{j+1} ; \beta_{i}\right)
\end{array} \\
& \begin{array}{lllll}
\left(\alpha_{1} ; \beta_{i+1}\right) & \left(\alpha_{2} ; \beta_{i+1}\right) & \ldots & \left(\alpha_{j} ; \beta_{i+1}\right) & \left(\alpha_{j+1} ; \beta_{i+1}\right)
\end{array}
\end{aligned}
$$

where $\beta_{i+1}-\beta_{i}=\Delta \beta$ и $\alpha_{i+1}-\alpha_{i}=\Delta \alpha$.

In fig. 2 , a and fig. 2 , b the interpolation grid of the twotier mechanism with the radius $R=500 \mathrm{~mm}, \Delta \alpha=10^{\circ}$ and $\Delta \beta=5^{\circ}$ is shown [3-10].

As seen in the fig.2,a and 2,b the specific arc-shaped curve is formed by the rod movement about the center in the point $O^{\prime}$ (fig. 1) in angle $\beta$. The set of arc-shaped curves is formed resulting from the rotation of the disk with the radius $R$ about the center at point $O$ in angle $\alpha$. The set of concentric circles with the center at the point $O$, forms as the result of the angles $\alpha$ and $\beta$ resolution. The set of cross-points of the concentric circles and the arc-shaped curves are the joint connections of the interpolation grid of the two-tier mechanism.

Let's locate a certain rectilinear segment in the twotier mechanism's operating area, with endpoints at $M 1(x 1 ; y 1)$ and $M 2(x 2 ; y 2)$. If solving the inverse kinematics at the point $M 1(x 1 ; y 1)$, we will have the values of the required parts rotation angles of the given mechanism which contain in the matrix (3). Such matrix element is denoted as $\left(\alpha_{j} ; \beta_{i}\right)$, where $0 \leq i<n$ и $0 \leq j<$ $m$. It is obvious, that $\left(\alpha_{j} ; \beta_{i}\right)$ in the matrix (3) is surrounded by nearby elements:

$$
\widehat{M}=\left(\begin{array}{ccc}
\ldots & \left(\alpha_{j} ; \beta_{i-1}\right) & \ldots \\
\left(\alpha_{j-1} ; \beta_{i}\right) & \left(\alpha_{j} ; \beta_{i}\right) & \left(\alpha_{j+1} ; \beta_{i}\right) \\
\ldots & \left(\alpha_{j} ; \beta_{i+1}\right) & \ldots
\end{array}\right)
$$

The summary of the method [2] is in the search for the nearby element from the matrix $\widehat{M}$ upon criterion of the minimal distance from such element's coordinate to the endpoint of the rectilinear segment $\overline{M 1 M 2}$. After such element is found, the search of its nearby elements upon the given criterion will be actualized again. Algorithm's iterative process will repeat until it will reach the end of the rectilinear segment $\overline{M 1 M 2}$.

\section{The result of computer simulation using the Brazenham algorithm}

In fig. 2 the result of the computer modelling in MATLAB is shown and it reflects the interpolation process of the rectilinear segment (line in fig. 2,a), indexed by two points, point $M 1[-350 ;-100]$ and point $M 2[160 ; 280]$. Interpolation is fulfilled according to the the least distance method using the two-tier mechanism with disk radius $R=500$ and $\Delta \alpha=10^{\circ}, \Delta \beta=5^{\circ}$.

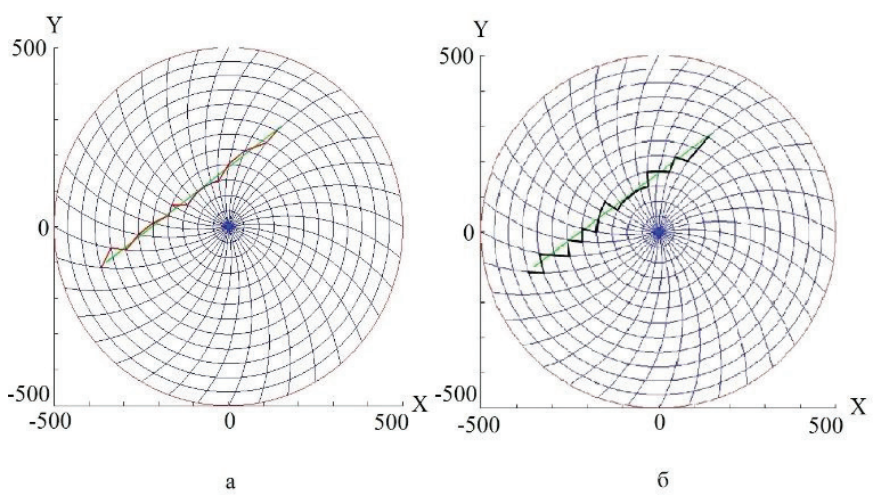

Fig.2. Results of the rectilinear segment interpolation: a - by the least distance method, b - Bresenham's algorithm

During the modelling it was discovered that rootmean-square deviation of the interpolation error by least distance method doesn't exceed the value of 9.914.

For the comparative analysis the rectilinear segment interpolation procedure by known Bresenham's algorithm was also actualized. The result of the computer modelling is presented in fig.2,b. This result was obtained with the same input parameters of the prototype [11-36].

\section{Conclusion}

Modelling showed that the root-mean-square deviation by the Bresenham's algorithm equals 15.799, which is significantly higher than the deviation with the least distance method, and this proves that the least distance 
method is expedient for the solving the interpolation problem in unorthogonal mechanisms.

\section{References}

1. Voltmander P.V. Mashinnaya grafika. Osnovnye algoritmy. [Computer graphics. Basic algorithms] (NGU, Novosibirsk 1997)

2. Valyukevich Y.A., Naumov I.I. Nauchnotehnicheskie vedomosti Sankt-Peterburgskogo gosudarstvennogo politehnicheskogo universiteta, no.95, 177-181 (2010) [in Russian]

3. V.E. Gough, S.G. Whitehall, In: Proc. 9th International Technical Congress, FISITA, 177 (1962)

4. D. Stewart, In Proc. Inst Mech. Eng., Pan I 180 (15) 371-386 (1966)

5. M. Valenti, Machine tools get smarter, ASME Mech. Eng. 70-75 (1995)

6. F. Pierrot, P. Dauchez, A. Fournier, J. Robotic Syst. No.8 829-840 (1991)

7. .F. Behi. IEEE J. Robotics Autom, no.4, 561-565 (1988)

8. R.I. Alizade, N.R. Tagiyev, J. Duffy, Mechanism and Machine Theory, no. 1, 115-124 (1994)

9. J.W. Kimetal., In: Proc. 1997 ASME IMECE Symposium on Machine Tools, (1997)

10. G.R. Dunlop, T.P. Jones, Mechanism and Machine Theory, no.8, 903-920 (1997)

11. J. Nielsen, B. Roth, Int. J. Robotics Res., no. 12, 1147 - 1160 (1999)

12. P. Nanua, K.J. Waldron, V. Murthy, IEEE Trans. Robotics Autom, no. 6, 438-444 (1990)

13. M.L. Musty, Mechanism and Machine Theory, no. 4 365-380

14. P. Dietmaier. Advances in Robot Kinematics: Analysis and Control1, 7-16 (1998)

15. Jack Bresenham. IBM Systems Journal, no.1, 25-30, (1965)

16. 14. Jack Bresenham. Communications of the ACM, no. 2, 100-106 (1977)

17. Jerry R. Van Aken, IEEE Computer Graphics \& Applications.24-35 (1984)

18. Michael Abrash. Dobbs Journal, no. 194, 171-176 (1992)

19. J.E. Bresenham, IBM Syst. J., no.4, 25-30 (1965)

20. J.E. Bresenham, Comput. J., no.25 116-120 (1982)

21.J.E. Bresenham, Fundamental Algorithms for Computer Graphics, 59-104 (1985)

22. D. Field, ACM Trans. Graph., 1-11 (1985)

23. H. Freeman, IRE Trans. EC-102, 260 -268 (1961)

24. H. Freeman, Picture Processing and Psychopictories, 241-266 (1970)
25. K.Y. Fung, T.M. Nicholl, A.K. Dewdney, Comput. Graph. Forum 3, 267-277 (1992)

26. G.W. Gill, IEEE Comput. Graph., 66 -72 (1994)

27. P. Graham, S. Iyengar,Iin: Proc. 1993 ACM Computer Science Conf. (1993)

28. P. Graham, S. Iyengar, IEEE Comput. Graph. Appl., 49-53 (1994)

29. G.B. Regiori, Digital computer transformations for irregular line drawings (Department of Electrical Engineering and Computer Science, New York 1972)

30. J. Rokne, B. Wyvill, X. Wu, ACM Trans. Graph, no. 9 (4) 376-388 (1990)

31. J. Rokne, C. Yao, ACM Trans. Graph., no.11(2) 183192 (1992)

32. A. Rosenfeld, IEEE Trans. Comput. $1264-1269$ (1974) 